\title{
Tissue factor expression in the metaplasia-adenoma-carcinoma sequence of gastric cancer in a European population
}

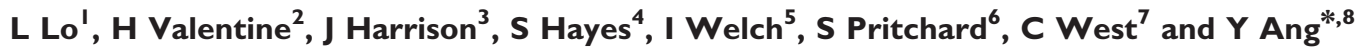 \\ 'Royal Perth Hospital, GPO Box X2213, Perth, Western Australia, 600 I; ${ }^{2}$ Translational Radiobiology Group, Institute of Cancer Sciences, Manchester \\ Academic Health Science Centre, The University of Manchester, Wilmslow Road, Manchester M20 4BX, UK; ${ }^{3}$ Department of Histopathology, Royal \\ Gwent Hospital, Newport, Wales, UK, NP20 2UB; ${ }^{4}$ Department of Histopathology, Salford Royal NHS Foundation Trust, Salford, M6 8HD, UK; \\ ${ }^{5}$ Department of Surgery, University Hospital of South Manchetser, Southmoor Road, Manchester M23 9LT, UK; ${ }^{6}$ Department of Histopathology Clinical \\ Sciences Building, University Hospital South Manchester, Southmoor Road, Manchester M23 9LT, UK; ${ }^{7}$ School of Cancer \& Enabling Sciences, \\ Manchester Academic Health Science Centre, The University of Manchester, Christie Hospital, Manchester, UK; ${ }^{8}$ Department Of Gastroenterology, Royal \\ Albert Edward Infirmary, Wigan Lane, Wigan, Greater Manchester WNI 2NN, UK
}

BACKGROUND: Tissue factor (TF), which has a role in normal tissue haemostasis, was reported to be aberrantly expressed, associated with higher microvascular density and a poor prognosis in intestinal-type gastric adenocarcinoma in the Japanese population. This is the first study to look at the relationship of TF and the metaplasia-adenoma-carcinoma sequence (MACS) of gastric cancer in a European population.

METHODS: The expression of TF was examined immunohistochemically in 19| gastric tissue samples: (|3: normal; |8: intestinal metaplasia; 160: gastric adenocarcinoma) from the European population.

RESULTS: TF was not expressed in normal gastric mucosal cells. A strong intensity of staining was found in intestinal metaplasia cells but in 2 of 18 samples. TF expression increased with advancing stage of gastric cancer $(P<0.000$ I, Jonckheere's test for ordered medians). Stage 3-4 gastric cancers preferentially expressed TF $(34 \%, P=0.04)$. In comparison with the Japanese study, TF was not expressed at a higher level in intestinal vs diffuse-type gastric cancers and expression had 'no prognostic' significance.

CONCLUSION: TF may be involved in tumour progression along the MACS of gastric cancer in the European population and is shown to start in precancerous lesions. However, clinical features may differ due to differences in biological features in the two populations, as reflected by differences in TF expression profile.

British Journal of Cancer (2012) 107, | |25-1 |30. doi: 10.1038/bjc.2012.363 www.bjcancer.com

Published online 28 August 2012

(c) 2012 Cancer Research UK

Keywords: tissue factor; gastric adenocarcinoma; metaplasia-adenoma-carcinoma sequence

Tissue factor (TF) is a transmembrane glycoprotein that is a major physiological initiator of blood coagulation. Its expression was detected in epithelial tissues of the skin, mucosal surfaces of the gut and genitourinary system (Drake et al, 1989). Abnormal expression of TF was reported in different types of cancers, including gastric (Yamashita et al, 2007), non-small cell lung (Koomagi and Volm, 1998; Sawada et al, 1999), breast (Vrana et al, 1996), pancreatic ductal (Nitori et al, 2005), colorectal (Kataoka et al, 1997; Shigemori et al, 1998; Seto et al, 2000; Nakasaki et al, 2002), hepatocellular (Poon et al, 2003) and prostate carcinoma (Abdulkadir et al, 2000).

A review in 2003 (Rickles and Fernandez, 2003) summarised its role in inducing angiogenesis-related metastasis to be both clotting-dependent and -independent. TF when bound to its natural ligand, FVIIa to form the TF:FVII complex activates antiapoptotic kinases, which could drive cancer metastasis (Sorensen et al, 2003). In baby hamster kidney cells, TF overexpression potently reversed hallmarks of apoptosis (Sorensen et al, 2003). FVIIa and TF also promote the survival of cells able to undergo

*Correspondence: Dr YS Ang; E-mail: Yeng.Ang@wwl.nhs.uk revised 25 June 20 I2; accepted 18 July 20 I2; published online 28 August 2012 anoikis (a special form of apoptosis when cells detach from extracellular matrix and lack adhesion signalling) (Versteeg et al, 2004). Hu et al (1999) designed an icon (immunoconjugate molecule) that localised selectively to TF-expressing cells. When injected into xenografted human prostate cancer and melanoma, it resulted in significant tumour debulking.

$\mathrm{TF}$ is also implicated in regulation of the synthesis of the pro-angiogenic vascular endothelial growth factor (VEGF) by tumour cells. When tumour cells were transfected to express TF in a murine xenograft model, they grew rapidly and established larger and more vascularised tumours than controls (Abe et al, 1999). Increased TF positivity has been correlated with increased vascular density, clinical stage and Duke's classification in colorectal cancers (Staton et al, 2007).

A relationship between elevated TF expression and poor prognosis in advanced-stage intestinal gastric cancer was shown in the Japanese population (Yamashita et al, 2007). In Japan, distal tumours tend to predominate in gastric cancers, in contrast to an increasing incidence of proximal gastric tumours in the West (Liu et al, 2004). The different distribution for the site of gastric cancers in these two populations may suggest separate biological entities and variations in respective TF expressions and clinical outcomes. This is the first study to examine TF expression in gastric 
adenocarcinoma in a European population. Correa proposed that the intestinal-type gastric cancer sequentially transformed from normal mucosa into atrophic gastritis, intestinal metaplasia, dysplasia and finally to invasive adenocarcinoma in a very similar fashion to the multi-step sequence of events from precancerous changes to invasive disease in colorectal cancer (Correa, 1988). This is subsequently supported by extensive studies by Keller et al (Keller et al, 2005), although controversies still exist. We also studied the expression of TF along carcinogenic metaplasiaadenoma-carcinoma sequence (MACS) of gastric cancer.

\section{MATERIALS AND METHODS}

\section{Patients and materials}

Specimens were obtained from the histopathology archive of the Department of Histopathology, South Manchester University Hospital NHS Trust and the Department of Pathology, Wigan Infirmary. The study was approved by the Tameside and Glossop Ethics Committee (03/TG/560). A retrospectively compiled database was established of 251 patients who underwent surgery for primary gastric and gastro-oesophageal tumours at the South Manchester University Hospital NHS Trust between 1995 and 2004. Certain groups of patients were excluded from the study, including those of Siewart type I $(n=22)$ and those who underwent neoadjuvant therapy $(n=31)$, emergency surgery $(n=1)$ or died after surgery $(n=25)$. Tissue microarrays (TMAs) were were available for 160 patients, 122 of whom were male, with median age of 67 (range $30-85$ ) years. The TNM seventh edition was used for all staging purposes. The 160 gastric cancer patients comprised stage $0(n=2)$, stage I $(n=27)$, stage II $(n=52)$, stage III $(n=68)$ and stage IV $(n=11)$ disease. The patients underwent either partial or subtotal gastrectomies $(n=38)$, total gastrectomy $(n=37)$, proximal gastrectomy $(n=4)$ or oesophago gastrectomy $(n=80)$ and completion gastrectomy $(n=1)$. After surgery, patients were seen in the surgical outpatient clinic. The median period for follow-up was 7.9 years (range: $4.0-13.3$ years). Thirteen normal tissue samples were available: 7 from males with mean age of 61 years. Eighteen intestinal metaplasia samples, of which 5 were from males with mean age of 68 years, were also included in this study (Table 1).

\section{Immunohistochemical staining of TF (CD142)}

Four tumour cores were available for each patient, of which two were from the centres and two from the invasive edges of the resected tumour. All specimens were stained within 2 months of cutting to minimise antigen deterioration. The polymeric method, more specifically the Super-Sensitive Detection System (Biogenex Laboratories, Fremont, CA, USA), which incorporates a 'Super Enhancer', to boost the effects of the polymeric-HRP secondary antibody was used. Sections $4-\mu \mathrm{m}$ thick were deparaffinised in xylene and rehydrated before microwaving in the $0.05 \mathrm{M} \mathrm{Tris-HCl} /$ 1 mM EDTA buffer ( $\mathrm{pH} 9.0$ ) for $25 \mathrm{~min}$ at high power. The sections were then allowed to cool in the buffer for $20 \mathrm{~min}$. After a 5 -min wash in Tris-buffered saline (TBS), blocking steps were followed according to the manufacturer's guidelines using hydrogen peroxide solution and $10 \%$ casein. After the casein solution was tapped off, Mouse Monoclonal Antibody no.4509 (American Diagnostica Inc., Stamford, CT, USA) diluted at 1:250 in TBS was applied and incubated in the humidified chamber for $1 \mathrm{~h}$ at room temperature. TBS washing was repeated and Biogenex Super Enhancer Solution was applied and sections were incubated for 20 min at room temperature. After washing with TBS, the Biogenex Polymer HRP Secondary was applied and sections were incubated for $30 \mathrm{~min}$ at room temperature. After washing, $\mathrm{DAB}+$ solution was applied at 1:50 dilution and the samples were left covered for $5 \mathrm{~min}$. Sections were then washed with water, counterstained with
Table I Patient demographics of samples examined for TF expression

\begin{tabular}{lccc}
\hline Sample & $\boldsymbol{n}$ & Male:female & Mean age \\
\hline Normal & 13 & $7: 6$ & 61 \\
Intestinal Metaplasia & 18 & $5: 13$ & 67.7 \\
Adenocarcinoma & 160 & $122: 58$ & 67 (range 30-85) \\
\hline
\end{tabular}

haematoxylin for $1 \mathrm{~min}$, alkaline dipped, dehydrated and mounted. Whole sections of 14 high and 14 low staining for TF were stained with CD31/CD34, markers of microvascular density (MVD). Antigen retrieval was carried out by microwaving for $25 \mathrm{~min}$ in $10 \mathrm{~mm}$ sodium citrate ( $\mathrm{pH}$ 6.0) (Sigma-Aldrich, Poole, UK) buffer solution. After quenching endogenous peroxidase, non-specific binding was blocked using 10\% casein solution (Vector Laboratories Inc., Peterborough, UK). A mixture of primary antibodies, mouse monoclonal anti-CD31 (M0823, Dako Ltd., Ely, UK) and anti-CD34 (M7165, Dako Ltd), both at dilution $1: 70$, was applied and the sections were incubated overnight at $4{ }^{\circ} \mathrm{C}$. After washing and application of biotinylated rabbit anti-mouse secondary antibody, detection was completed using Vectastain ABC-AP Kit (SK-5100, Vector) according to the manufacturer's instructions using Vector Red (SK-5000, Vector) chromogen and haematoxylin counterstain.

\section{Assessment of TF staining in the gastric cancer samples}

Staining for TF was isolated to the cytoplasm and basement membrane of expressing cells. Only tumour cells were scored as positive. Lymphocytes, mast cells, parietal and chief cells at the basal pits of the normal gastric mucosa were also prone to strong staining but were not scored. Both the intensity and percentage of staining were scored. The intensity was scored on a scale from 0 to 4 with 0 for negative uptake, 1 for weak, 2 for moderate, 3 for strong and 4 for very strong staining. Heterogeneous staining and slight background staining was noted in most of the TMA core samples studied. In a sample where there was weak, moderate and strong staining in different proportions, the strongest staining and its percentage was recorded. The immunoreactivity of $\mathrm{TF}$ was evaluated as positive when the intensity score was $\geqslant 3$ and $>10 \%$ of the carcinoma cells were stained in the case of assessing for the strongest intensity. The intensity for the majority of tumour cells stained and its percentage were also recorded for further analysis. Assessment of immunoreactivity was performed by an individual (LL) without background knowledge of the specimens and then assessed by another team member ( $\mathrm{SH})$. Inter-observer variability was $<23 \%$.

\section{Statistical analysis}

Data were analysed using S-Plus 2000 and appropriate nonparametric tests were used to investigate TF expression and clinicopathological parameters (Wilcoxon rank sum test and Chisquare test for two independent groupings and Jonckheere's test for ordered medians (JTOM)). As there was a maximum of four scores for TF expression available for each patient, the mean scores were taken for statistical analysis. The scores for intensity $(I)$ and percentage $(P)$ were substituted in a formula to obtain a scale. The formula was decided to be $100^{\star} I_{s}+P_{s}$, which produced a scale ranging from 0-500 for $\mathrm{TF}$ expression in terms of strongest intensity $\left(I_{\mathrm{s}}\right)$. A score of $>310$ was used as a cut-off for positivity. The cut-off was chosen in order to mimic the criteria for positivity used by Yamashita et al (2007). Scores based on the intensity $\left(I_{\mathrm{m}}\right)$ of the majority of cancer cells stained were also recorded and the formula $I_{\mathrm{m}}{ }^{\star} P_{\mathrm{m}}$ was used to obtain a scale of $0-400$ for tissue expression. This scale was compared against clinicopathological parameters to observe for trend differences in comparison with the ones when strongest intensity $\left(100^{\star} I_{\mathrm{s}}+P_{\mathrm{s}}\right)$ was emphasised in 

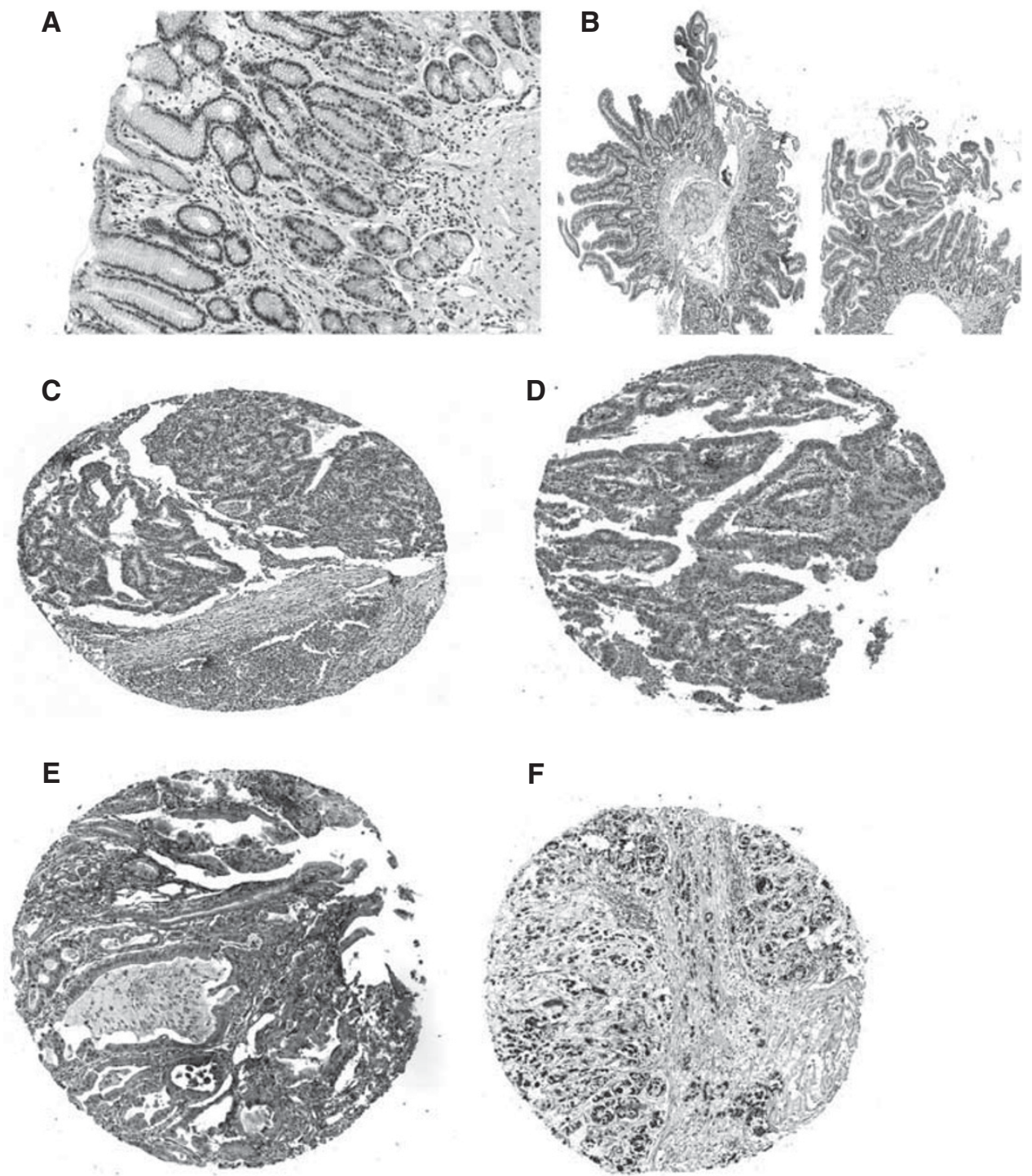

Figure I (A) Normal gastric tissue with negative TF staining. (B) Intestinal metaplasia with strong TF staining at edges of ductal structures. (C) Weak staining (score I (I); 100\% (P)) of gastric cancer cells. (D) Moderate staining (score $2(I)$; I00\% (P)) in gastric cancer cells. (E) Strong staining (score 3 (I); $80 \%$ $(P))$ in gastric cancer cells. $(\mathbf{F})$ Very strong staining (score $4(I) ; 100 \%(P))$ in gastric cancer cells.

scoring the specimens. The Kaplan-Meier method was used to compare the survival data of 160 patients to TF expression. Survival time was measured as the time from the date of surgery until death or last follow-up appointment. The significance of the difference between the variables was calculated by a Log-rank test on these data.

\section{RESULTS}

\section{Immunohistochemical staining of TF in gastric cancer}

In normal mucosa, moderate to strong staining (scores 2 and 3 ) was seen in the parietal and chief cells at the basal pits. There was an associated mixed chronic inflammatory cell infiltrate (lymphocytes/mast cells) in the submucosa that also stained strongly. None of the ductal structures in the normal gastric samples $(n=13)$ stained. As only ductal structures were relevant, all normal tissue was considered negative for TF expression. Only 2 out of the 18 intestinal metaplasia samples stained positive for $\mathrm{TF}(\mathrm{TF}>310)$ but, when staining was present, it was at a high intensity. The staining intensity of intestinal metaplasia samples ranged from score 1 to 3 , with a majority having score 3 , but with a small
Table 2 Increasing TF expression along the gastric adenoma-carcinoma sequence

\begin{tabular}{lcc}
\hline Tissue type & Patients & TF positive $^{a}$ \\
\hline Normal mucosa & $0 / 13$ & $0 \%$ \\
Intestinal metaplasia & $2 / 18$ & $11 \%$ \\
Stage 0 & $0 / 2$ & $0 \%$ \\
Stage 1 & $5 / 27$ & $19 \%$ \\
Stage 2 & $11 / 52$ & $21 \%$ \\
Stage 3 & $21 / 68$ & $31 \%$ \\
Stage 4 & $6 / 11$ & $55 \%$ \\
\hline
\end{tabular}

Increasing expression with gastric cancer progression was significant with Jonckheere's test for ordered medians; $P=0.0005$. ${ }^{a}$ Positive expression $=100 * I_{s}+P_{s}>310$

percentage, mean $6 \%$ and range $0-15 \%$. Staining was seen in tumour-cell cytoplasm and cell membrane. The staining pattern was heterogeneous in intensity and percentage of stained cells. The staining intensity was relatively concentrated in signet ring cells and carcinoma cells situated near the vessel endothelium. In all, 43 out of $160(27 \%)$ tumours were classified positive for TF. The other 


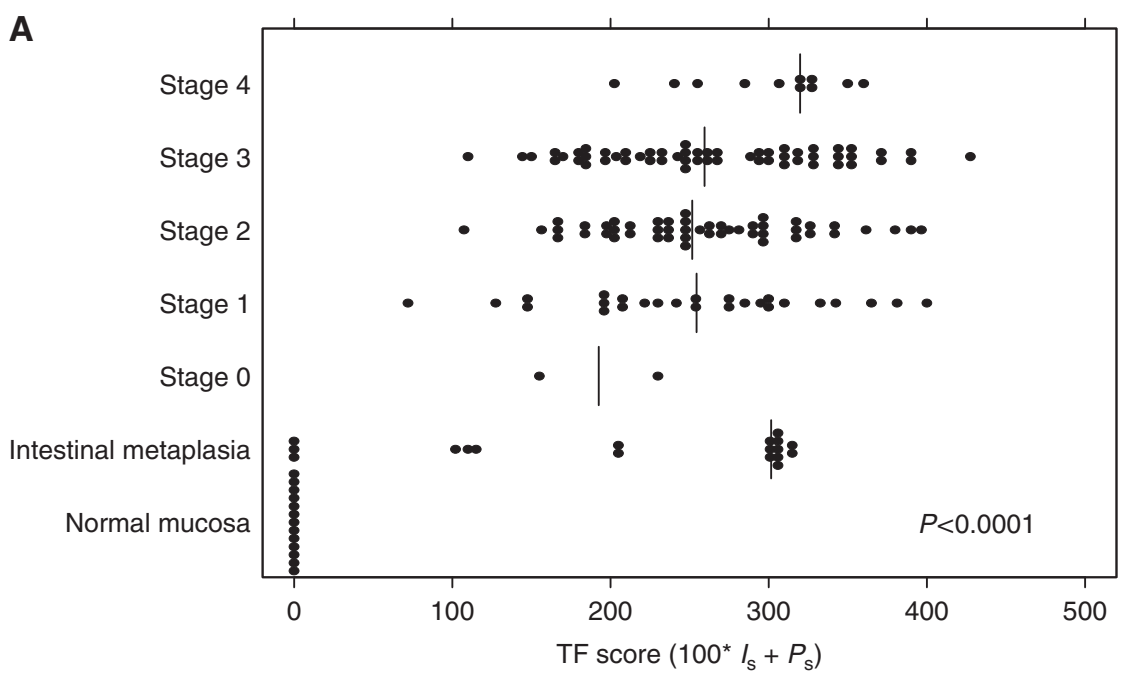

B

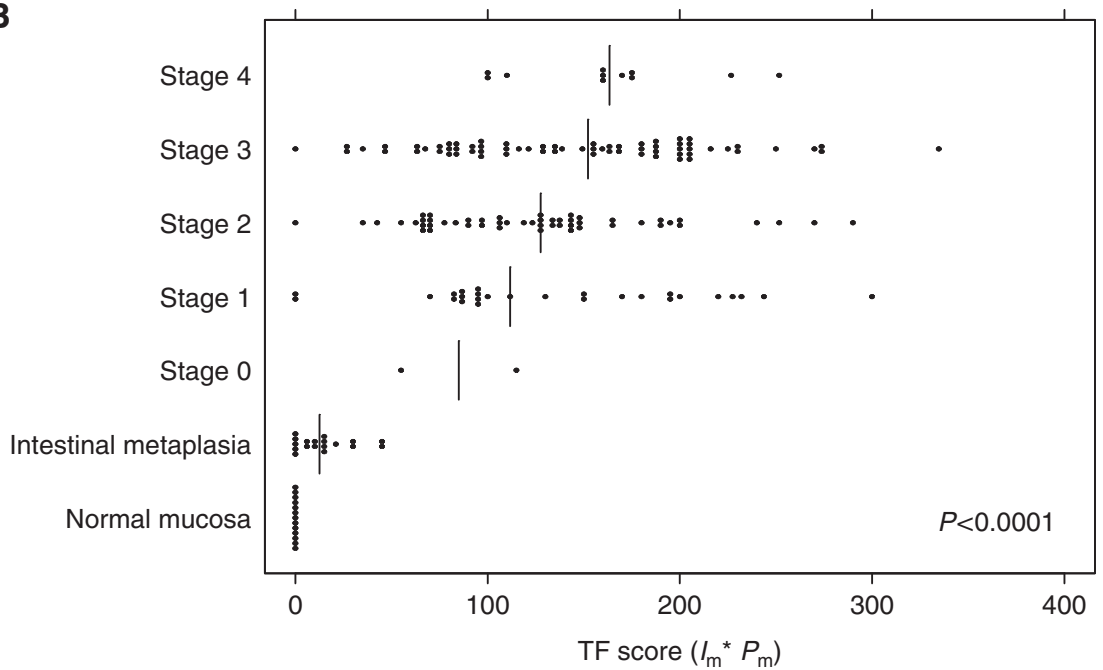

Figure 2 (A) Increasing TF expression with $\left(100 * I_{s}+P_{s}\right)$ scale across the gastric adenoma-carcinomic sequence with JTOM; $P<0.0001$. (B) Increasing TF expression with $\left(I_{m}{ }^{*} P_{m}\right)$ scale across the gastric adenoma-carcinomic sequence with Jockheere's test for ordered medians; $P<0.000 I$.

117 cases $(73 \%)$ demonstrated a staining intensity that was weaker than that found in the majority of intestinal metaplasia samples, but with a much higher percentage of cells being stained and these were classified as TF negative (Figure 1A-F). All negative controls showed no immunoreactivity. There was a trend for increased TF positivity along the adenoma-carcinoma sequence. (Table 2; Figure 2). The increasing trend for TF expression against overall stage $0-4$ gastric adenocarcinoma was more significant for the alternative scoring method $(P=0.01$; (Figure $2 \mathrm{~A})$ when compared with the $I_{\mathrm{m}}$ scale $(P=0.025$; Figure $2 \mathrm{~B}$ ) by JTOM. Moreover, by taking into account the normal and intestinal metaplasia tissue to examine TF expression along the gastric MACS, the increasing trend was shown to be highly significant in both approaches; $P<0.0001$ by JTOM for both $I_{\mathrm{s}}$ and $I_{\mathrm{m}}$ scales (Figure 2 ). $P$ value was more significant in the approach where $I_{\mathrm{m}}$ and $P_{\mathrm{m}}$ of stained cells were recorded. However, trends of increase in TF expression were not found to be significant by JTOM with other clinicopathological features (tumour differentiation, residual resection margins, $\mathrm{T}$ staging, $\mathrm{N}$ staging and $\mathrm{M}$ staging) in both approaches. The was no statistically significant difference in MVD of gastric adenocarcinoma with positive $(n=25)$ vs negative $(n=25)$ TF expression.

\section{Clinicopathological features and TF expression}

The association between TF expression and clinicopathological parameters is presented in $100^{\star} I_{s}+P_{s}$ The above formulae and a cut-off of 310 was chosen to produce results that could be compared with the findings in Yamashita et al (2007), where gastric cancer cells were scored as TF positive when $>10 \%$ of cells stained intensely.

No significant differences were detected between positive $\mathrm{TF}$ expression and most of the clinicopathological features (gender, location, histological appearance, $\mathrm{T}$ staging, $\mathrm{N}$ staging and $M$ staging), with the exception of overall TNM staging. The difference, tested by Chi-square test was significant when the overall TNM staging was grouped as $0-2$ vs 3-4, $P=0.04$. This supports the finding of an ordered increase in $\mathrm{TF}$ expression from stage 0 to stage $4, P=0.01$. The differences in $\mathrm{N}$ staging had borderline significance, $P=0.054$, with $31 \%$ of patients having positive lymph-node status compared with $19 \%$ patients with negative lymph-node status expressing $\mathrm{TF}>310$. Similar trends for TF expression against clinicopathological parameters were seen when using the $I_{\mathrm{m}}\left(I_{\mathrm{m}}{ }^{\star} P_{\mathrm{m}}\right)$ method.

In Lauren subtypes, no significant relationship was found between positive $\mathrm{TF}$ expression $(\mathrm{TF}>310)$ and clinicopathological parameters. However, a borderline significant trend was seen for a relationship between TF expression and T $(P=0.07)$ and $\mathrm{N}$ stage $(P=0.06)$ for diffuse-type gastric adenocarcinoma. The was no relationship between TF expression and prognosis (Figure 3A-D). 
A
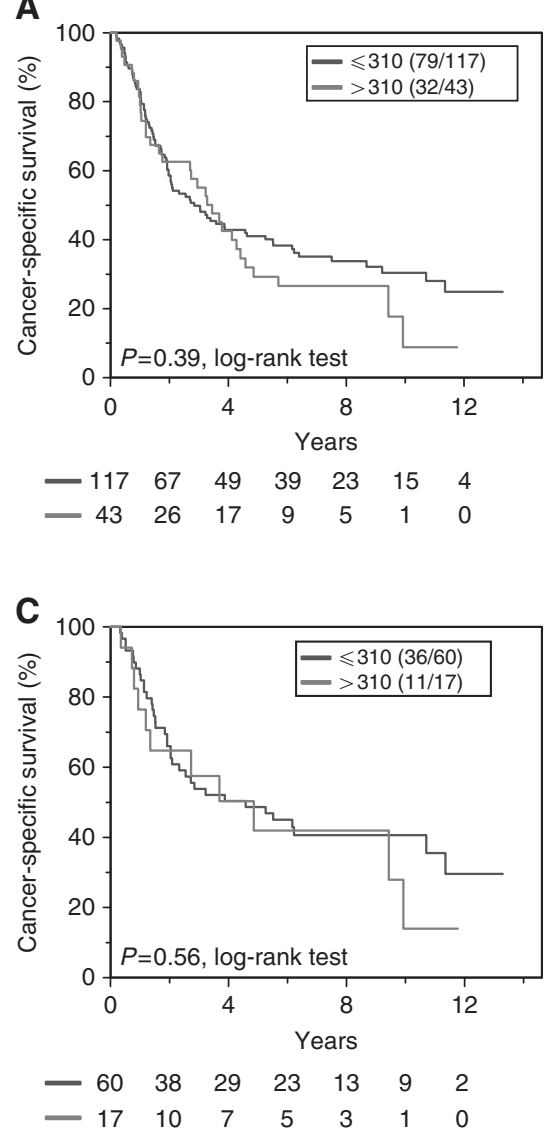

B

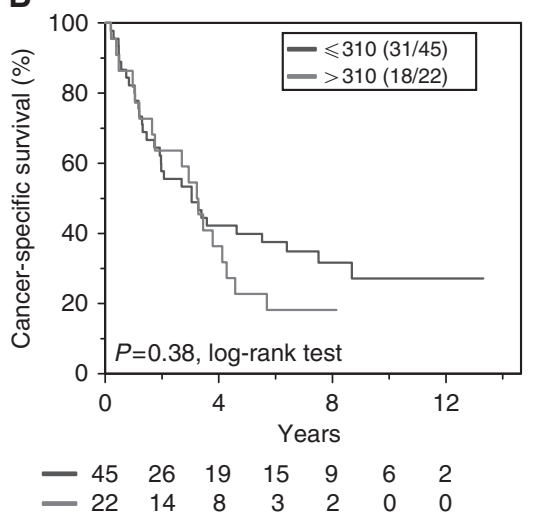

D

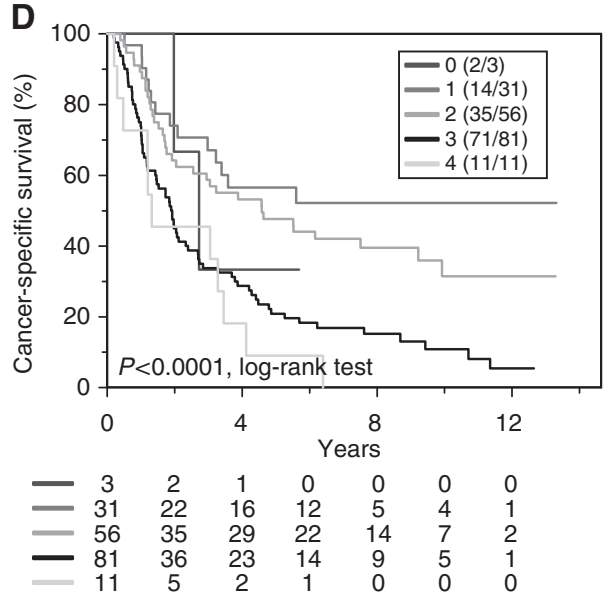

Figure 3 (A) Survival outcome in TF positive (TF>310) cancers. (B) Survival outcome in diffuse-type TF-positive cancers. (C) Survival outcome in intestinal-type TF-positive cancers. (D) Survival outcome in patients with Stage 0-4 gastric adenocarinoma.

\section{DISCUSSION}

TF expression and its relationship with treatment outcome in gastric adenocarcinoma has been reported previously in Japan (Yamashita et al, 2007). Our report is the first study to look at TF expression along the MACS of gastric adenocarcinoma and to investigate the relationship with prognosis in a European population. In this study, immunohistochemistry involved recording both the strongest intensity of staining $\left(I_{\mathrm{s}}\right)$ and the intensity for the majority of cells stained $\left(I_{\mathrm{m}}\right)$ and their respective percentages to study the influence of different scoring methods on the relationship between $\mathrm{TF}$ expression and outcomes. With the $100^{\star} I_{\mathrm{s}}+P_{\mathrm{s}}$ scale (Figure $2 \mathrm{~A}$ ), the intestinal metaplasia gastric cells exhibit a higher peak in positive TF expression (TF $>310)$ than those in stage 0 cancer. In the $I_{\mathrm{m}}{ }^{\star} P_{\mathrm{m}}$ scale, TF expression of intestinal metaplasia cells would still record a lower value than stage 0 cancer, giving a smooth increasing trend for TF expression. Although found in many studies (Kösem et al, 2009; Liu et al, 2010), we were unable to confirm the high MVD in high TF-expressing tumours. This may be due to the intrinsic biological difference or different staging systems between European and East Asian gastric cancers. There are main differences in distribution of gastric cancers in these two hemispheres, which include tumour location, with more distal or middle thirds involvement in Japanese patients, and Lauren subtypes, predominantly the intestinal type in Japan. Our study has shown that TF expression was present in intestinal metaplasia gastric cells, with stronger TF staining intensities but lower percentages compared with lower stage cancers.
There are very similar trends in TF expression with clinicopathological parameters in both our and the previously reported approaches. With the scale that placed emphasis on intensity of staining $\left(100^{\star} I_{\mathrm{s}}+P_{\mathrm{s}}\right)$, a more significant result was found, $P=0.01$, for the increasing trend of TF expression in stages $0-4$ gastric adenocarcinoma. Looking at the adenoma-carcinoma sequence on the whole, the increasing trend was very significant, $P<0.0001$, for the TF expression from normal tissue, intestinal metaplasia and throughout all stages of gastric adenocarcinoma. A similar outcome was found in the $I_{\mathrm{m}}{ }^{*} P_{\mathrm{m}}$ approach. The increasing trend in the $I_{\mathrm{m}}{ }^{\star} P_{\mathrm{m}}$ scale indicates that both intensity and percentage of TF expression increase with stages of gastric cancer. When the results were narrowed down to cut-off values and into subdivided groupings of clinicopathological parameters, there are different outcomes for two scales in the aspect of overall TNM staging. A slight significant difference, $P=0.04$ was found with the $100^{\star} I_{\mathrm{s}}+P_{\mathrm{s}}$ scale rather than with the $I_{\mathrm{m}}{ }^{*} P_{\mathrm{m}}$ scale, $P=0.24$. Difference in outcome might reflect the impact of intensity of TF staining being stronger compared with percentage staining in determining the relationship with overall TNM staging. It also reflects that the different approach in scoring can influence outcome.

This study has not been able to confirm a higher TF expression in intestinal-type cancers, found in Yamashita et al (2007) (28\% intestinal-type and 33\% diffuse-type cancers). Diffuse-type cancers are known to be more invasive and have a poorer prognosis compared with intestinal types. TF has been related to cancer metastasis in many studies; its relationship with invasiveness could be highlighted in this study with the slightly 
higher proportion of diffuse-type cancers being TF positive. There was no significant difference found between $M$ staging and positive TF expression in our gastric cancer series. We were unable to confirm the significant relationship between $\mathrm{TF}$ expression and survival specifically in intestinal-type gastric cancer, as demonstrated in the Japanese group. Although a significant increase in TF expression was found with advancing stage, which is a good predictor of survival outcome, no relationship was seen. The huge variation in TF expression across all the stages of gastric cancer suggests that factors other than TF are influencing survival. As with the different outcomes with the roles of TF and FVIIa in signal transduction in different cell types, there may be a genetic variation in the propensity for TF expression in gastric cancer cells of different individuals of the same population. This is supported by various studies concluding in the nature of FVIIa:TF-induced signalling as being variable and cell-type-dependent (Cunningham et al, 1999; Camerer et al, 2000; Wang et al, 2002; Versteeg et al, 2004). As TF could act as a receptor for FVIIa in activating many cellular pathways, further work could be done to research the role of FVIIa and their relationship with VEGF and MVD. This would help us determine whether TF or FVIIa could cause angiogenic switch early on before the gastric cancer stages.

\section{REFERENCES}

Abdulkadir SA, Carvalhal GF, Kaleem Z, Kisiel W, Humphrey PA, Catalona WJ, Milbrandt J. Tissue factor expression and angiogenesis in human prostate carcinoma. Hum Pathol (2000) 31: 443-447

Abe K, Shoji M, Chen J, Bierhaus A, Danave I, Micko C, Casper K, Dillehay DL, Nawroth PP, Rickles FR. Regulation of vascular endothelial growth factor production and angiogenesis by the cytoplasmic tail of tissue factor. Proc Nat Acad Sci USA (1999) 96(15): 8663-8668

Camerer E, Huang W, Coughlin SR. Tissue factor- and factor X-dependent activation of protease-activated receptor 2 by factor VIIa. Proc Nat Acad Sci USA (2000) 97(10): 5255-5260

Correa P. A human model of gastric carcinogenesis. Cancer Res (1988) 48(13): 3554-3560

Cunningham MA, Romas P, Hutchinson P, Holdsworth SR, Tipping PG. Tissue factor and factor VIIa receptor/ligand interactions induce proinflammatory effects in macrophages. Blood (1999) 94: 3414-3420

Drake TA, Morrissey JH, Edgington TS. Selective cellular expression of tissue factor in human tissues. Implications for disorders of hemostasis and thrombosis. Am J Pathol (1989) 134: 1087-1097

$\mathrm{Hu}$ Z, Sun Y, Garen A. Targeting tumor vasculature endothelial cells and tumor cells for immunotherapy of human melanoma in a mouse xenograft model. Proc Nat Acad Sci USA (1999) 96(14): 8161-8166

Kataoka H, Uchino H, Asada Y, Hatakeyama K, Nabeshima K, Sumiyoshi A, Koono M. Analysis of tissue factor and tissue factor pathway inhibitor expression in human colorectal carcinoma cell lines and metastatic sublines to the liver. Int J Cancer (1997) 72(5): 878-884

Keller G, Hofler H, Becker KF. Molecular medicine of gastric adenocarcinomas. Expert Rev Mol Med (2005) 7(17): 1-13

Koomagi R, Volm M. Tissue-factor expression in human non-small-cell lung carcinoma measured by immunohistochemistry: correlation between tissue factor and angiogenesis. Int J Cancer (1998) 79: 19-22

Kösem M, Tuncer I, Kotan C, Ibiloğlu I, Oztürk M, Türkdoğan MK. Significance of VEGF and microvascular density in gastric carcinoma (2009) Hepatogastroenterology 56(93): 1236-1240

Liu N, Zhang LH, Du H, Hu Y, Zhang GG, Wang XH, Li JY, Ji JF (2010) Overexpression of endothelial cell specific molecule-1 (ESM-1) in gastric cancer. Ann Surg Oncol 17(10): 2628-2639

Liu Y, Kaneko S, Sobue T (2004) Trends in reported incidences of gastric cancer by tumour location, from 1975 to 1989 in Japan. Int J Epidemiol 33: $808-815$

Nakasaki T, Wada H, Shigemori C, Miki C, Gabazza EC, Nobori T, Nakamura S, Shiku H (2002) Expression of tissue factor and vascular
In conclusion, this study found no TF expression in normal gastric mucosal cells. A strong intensity staining was found in intestinal metaplasia cells but in a small percentage of samples. We confirmed that TF expression increases with advancing stage of gastric cancer. In contrast to previous Japanese study, findings in this Western population support a slightly higher proportion of positive TF expression in diffuse subtypes, correlating with their more invasive behaviour. Different approaches to scoring could potentially affect outcomes in studies that involve immunohistochemical staining. An approach that utilises the strongest intensity score observed in specimens and its percentage seems to give a significant result for positive expression ( $\mathrm{TF}>310)$ in influencing overall TNM staging. TF expression also displays a huge variation across all gastric cancer stages in different individuals of the same population and is not significantly associated with survival outcome.

\section{ACKNOWLEDGEMENTS}

YS Ang receives support from the UK NIHR/UKCRN (UK National Institute of Health Research/Cancer Research Network) and Research and Development Department of Wrightington Wigan and Leigh NHS Foundation Trust. C West is supported by the UK Experimental Cancer Medicine Centre Funding. endothelial growth factor is associated with angiogenesis in colorectal cancer. Am J Hematol 69: 247-254

Nitori N, Ino Y, Nakanishi Y, Yamada T, Honda K, Yanagihara K, Kosuge T, Kanai Y, Kitajima M, Hirohashi S (2005) Prognostic significance of tissue factor in pancreatic ductal adenocarcinoma. Clin Cancer Res 11: 2531-2539

Poon RT, Lau CP, Ho JW, Yu WC, Fan ST, Wong J (2003) Tissue factor expression correlates with tumor angiogenesis and invasiveness in human hepatocellular carcinoma. Clin Cancer Res 9: 5339-5345

Rickles FRPS, Fernandez PM (2003) Tissue factor, Thrombin, and Cancer. Chest 124: 58-68

Sawada M, Miyake S, Ohdama S, Matsubara O, Masuda S, Yakumaru K, Yoshizawa Y (1999) Expression of tissue factor in non-small-cell lung cancers and its relationship to metastasis. Br J Cancer 79(3-4): 472-477

Seto S, Onodera H, Kaido T, Yoshikawa A, Ishigami S, Arii S, Imamura M (2000) Tissue factor expression in human colorectal carcinoma: correlation with hepatic metastasis and impact on prognosis. Cancer 88(2): 295-301

Shigemori C, Wada H, Matsumoto K, Shiku H, Nakamura S, Suzuki H (1998) Tissue factor expression and metastatic potential of colorectal cancer. Thromb Haemost 80(6): 894-898

Sorensen BB, Rao LV, Tornehave D, Gammeltoft S, Petersen LC (2003) Antiapoptotic effect of coagulation factor VIIa. Blood 102(5): 1708-1715

Staton CA, Chetwood AS, Cameron IC, Cross SS, Brown NJ, Reed MW (2007) The angiogenic switch occurs at the adenoma stage of the adenoma carcinoma sequence in colorectal cancer. Gut 56(10): 1426-1432

Versteeg HH, Spek CA, Richel DJ, Peppelenbosch MP (2004) Coagulation factors VIIa and Xa inhibit apoptosis and anoikis. Oncogene 23(2): 410-417

Vrana JA, Stang MT, Grande JP, Getz MJ (1996) Expression of tissue factor in tumor stroma correlates with progression to invasive human breast cancer: paracrine regulation by carcinoma cell-derived members of the transforming growth factor beta family. Cancer Res 56: 5063-5070

Wang X, Gjernes E, Prydz H (2002) Factor VIIa induces tissue factordependent up-regulation of interleukin- 8 in a human keratinocyte line J Biol Chem 277(26): 23620-23626

Yamashita H, Kitayama J, Ishikawa M, Nagawa H (2007) Tissue factor expression is a clinical indicator of lymphatic metastasis and poor prognosis in gastric cancer with intestinal phenotype. J Surg Oncol 95: 324-331

This work is published under the standard license to publish agreement. After 12 months the work will become freely available and the license terms will switch to a Creative Commons Attribution-NonCommercial-Share Alike 3.0 Unported License. 